\title{
The Direction of Time and the Dynamical Evolution of the World
}

\author{
Hans H. Diel* \\ Diel Software Entwicklung und Beratung, Seestr. 102, 71067 Sindelfingen, Germany \\ *Corresponding author: diel@netic.de
}

Received December 08, 2014; Revised January 05, 2015; Accepted January 08, 2015

\begin{abstract}
The possible identification of an arrow of time with the dynamical evolution of the world, based on the laws of physics and without recourse to the flow of entropy, is investigated. It is concluded that when, in addition to their declarative semantics, the laws of physics are considered in the context of their overall goal, namely, describing the dynamical evolution of the world, a direction of time becomes apparent.
\end{abstract}

Keywords: causality, laws of physics, direction of time, time arrow, time symmetry

Cite This Article: Hans H. Diel, “The Direction of Time and the Dynamical Evolution of the World.” International Journal of Physics, vol. 3, no. 1 (2015): 12-16. doi: 10.12691/ijp-3-1-3.

\section{Introduction}

Much has been written (for example $[6,8,11,15]$ ) in regard to the (possible) identification of a direction of time associated with the dynamical evolution of the world. Typically, the discussion starts with the statement that the laws of physics are symmetric under a reversal of the direction of time. Observations that give the impression of the existence of a direction of time are then explained as a consequence of an increase in entropy as stated by the second law of thermodynamics, or "entropy law". Thus, the existence of an arrow of time is established, but it is implied purely from the entropy law. This approach can be considered unsatisfactory, for the following reasons:

- The entropy law is statistical in nature.

- It is not necessarily clear whether the entropy law is a fundamental law of physics or a consequence of other, more fundamental laws.

- The statement that the laws of physics are symmetric under a reversal of the direction of time is imprecise and therefore open to scrutiny. In some areas of physics (mainly quantum theory), it is also questionable whether the claimed symmetry can be defended.

This paper investigates whether it may be possible to identify a direction of time based on the laws of physics aside from the entropy law. In this pursuit, the laws of physics are analyzed in the context of their application within the dynamical evolution of the world. Five subjects are investigated in more detail in what follows:

- Time symmetry within the laws of physics - Because the most prominent argument against an arrow of time is the apparent symmetry of the laws of physics under the reversal of time, a critical view of this symmetry is deemed necessary.

- The laws of physics per se - The temporal symmetry claimed by some physicists and philosophers refers to the laws of physics. This section discusses what the laws of physics should encompass to represent a suitable basis for a discussion of time symmetry.

- The relationship between causality, time, and the arrow of time - A proper discussion about an arrow of time requires at least some very general considerations of time and causality.

- An arrow of time within the world's overall dynamical evolution -Even when time symmetry within the laws of physics can be negated (section 2), this does not necessarily imply a direction of time; arguments for such a direction still have to be identified. It is here claimed that possible arguments have to consider the world's overall dynamical evolution process.

- The observation of time symmetry / asymmetry - If a direction of time were not observable, theoretical arguments for its identification would be highly suspect.

\section{Time Symmetry}

\subsection{What Does Symmetry with Respect to Time Mean?}

In [10], page 687, R. Penrose writes "... despite of the fact that the dynamical and classical mechanics are symmetrical under a reversal of the direction of time!". He explains this further by stating "As far as mathematics is concerned, one can just as well specify final conditions, at some remote future time, and evolve backwards in time.".

This definition of "symmetrical under a reversal of the direction of time" seems to be the agreed upon understanding of physicists and philosophers who discuss the possible existence of an arrow of time $[6,8,11,15])$. Therefore we summarize: 
A law of physics is considered to be symmetrical under a reversal of the direction of time if, instead of starting from initial conditions to determine the future, one can just as well specify final conditions, at some remote future time, and evolve backwards in time.

Notice the phrasing "can just as well specify" in the above definition. It means that the predicate "symmetrical under a reversal of the direction of time" is first of all a predicate which applies to the functions used to describe the dynamical evolution of the systems. It does not, in fact, necessarily mean that the dynamical evolution at any point requires or allows the application of the time reversed functions. The application of the functions has to be discussed on top of the nature of the functions. Within mathematics, functions with the above described property are called bijective functions (see [2]). Thus, in mathematical terms the definition of "symmetrical under a reversal of the direction of time" is that the functions that represent the laws of physics are bijective functions.

\subsection{Are the Laws of Physics Time Symmetrical?}

For the purpose of this section, let us assume the laws of physics are reflected in a collection of functions to be used to compute the dynamical evolution of a physical system. The question then is whether all these functions are bijective functions. In other words, does there exist an inverse function that delivers the initial parameters when applied to the function results for each of these functions?

\subsubsection{Classical Mechanics}

Without going into detail, it is a generally agreed upon assumption that the laws of classical mechanics are symmetric under a reversal of the direction of time.

\subsubsection{Quantum Theory}

Quantum theory requires that every operator be unitary, which means that for each operator $U$, there must exist an inverse operator $U^{\dagger}$ such that $U U^{\dagger}=1$, i.e., such that the adjoint $U^{\dagger}=\mathrm{U}^{-1}$.

Thus, the operators of quantum physics may be considered "time symmetric." However, these time-symmetric operators cover only the normal (so-called unitary) development of the wave function. The measurement process is suspected to stop the unitary progression of the wave function and represent a non-unitary function (see [9]). Attempts to explain the measurement process as a continued unitary progression of the wave function (see $[3,15])$ do not seem to be generally accepted among physicists.

As an additional asymmetry in quantum theory, one may consider the laws of quantum field theory (QFT) dealing with interactions (e.g., scatterings) between particles. QFT provides methods for the determination of the scattering matrix $\mathrm{S}$, which enables the computation of the evolution of the wave functions $\psi_{1}$ and $\psi_{2}$ of two particles P1 and P2 toward a common wave function $\psi_{\mathrm{r}}$ representing the (possible) scattering results, $\left|\psi_{r}\right\rangle=S\left|\psi_{1}, \psi_{2}\right\rangle$. Although proofs for the unitarity of the S-matrix are given in text books of QFT (see, for example [14], p. 113))), it is questionable whether this indeed demonstrates the reversibility of the scattering process when $\left|\psi_{r}\right\rangle$ is an entangled state and $\left.\psi_{1}, \psi_{2}\right\rangle$ is a product state.

\subsubsection{Relativity Theory}

In [10], p. 409, R. Penrose mentions "some new issues that are raised by general relativity" in the context of black holes concerning time symmetrical dynamic determinism. It is not yet clear whether these new issues may result in the need for non-symmetrical laws or if existing laws might be found to be non-symmetrical.

\section{The Laws of Physics}

Statements about symmetries are typically abstractions; they hold true only if certain aspects of reality are ignored. Therefore, in mathematics and physics, the aspects and objects to which a symmetry applies are precisely defined. The subject of this section, "symmetry under reversal of time," applies to the "laws of physics." What precisely belongs, or should belong, to the laws of physics is usually not further addressed in discussions of the subject "symmetry under reversal of time." For a general discussion of the typical properties of the equations and functions used to express the laws of physics, a relatively vague understanding of what makes up those laws may be sufficient. However, when an (apparent) symmetry of the laws of physics is used to deny the existence of a direction of time, it is best to ensure that the relevant set of laws is properly understood.

What exactly are the laws of physics, and what do they mean, explicitly and implicitly? The subject is complex, as well as controversial. ${ }^{1}$ Here it is contended that, whatever a generally agreed upon opinion of the subject may be, the laws of physics should at least include some rules, i.e., some type of metalaws, regarding

1. The overall process of world-state progression; and

2. The difference between calculating future states and past states.

Many physicists would probably argue that such rules are implicitly provided within the existing theories of physics. Assuming this to be true, these implicit rules should be stated explicitly. If, on the other hand, the existence of such rules and their very need, were to be denied, physicists would give up an essential part of their (presumed) objectives, responsibilities, or both.

For a proper discussion of the laws of physics, it is also useful to discuss their purpose and application. Two main types of applications are apparent:

1. The laws of physics are applied by human beings (including through computers programmed by human beings) to compute (a) the state S1 that will evolve from state S0, and (b) the state S0 whose evolution resulted in state $\mathrm{S} 1$. Case a may be considered equivalent to computing a future state, and case b to computing a past state. In the next section, case a is said to apply the "forward computation" version of the respective laws, and case b the "backward computation."

2. The laws of physics are applied by nature to determine the continuous evolution of our world. Put the other way around, the laws of physics have been established with the goal of correctly describing the method by which nature determines the continuous evolution of the world.

${ }^{1}[5]$ provides an extensive analysis of the subject. 
Note that nature exclusively uses the forward computation version of the functions. Any claimed symmetry between the forward and backward computations (if it exists at all) is broken by the unilateral application of the laws of physics by nature. ${ }^{2}$

In sections 4 and 5, these relationships are discussed in more detail.

\section{Causality, Time, and the Direction of Time}

"The direction of time is deeply rooted in our causality thinking” (translated from German by the author) writes H.-D. Zeh in [15]. The concepts of time and causality are closely related. In the following, the relationship between causality, time, and the arrow of time is discussed. To facilitate this, a completely deterministic world is considered. ${ }^{3}$

The model suggested thus allows both causality and the direction of time. However, this should not be viewed as an a priori assumption of causality. To discuss the validity of causality and the direction of time, these concepts need to be introduced before their necessity may, in a second step, be verified.

\subsection{Causality}

For the discussion on what causality means, we will initially avoid any reference to time or related terms. The relation between causality and time will then be discussed in a second step.

For the first step (discussing what causality means), we need to introduce "states". We assume that the laws of physics are formulated in terms of states of the world. The laws describe how a state is a consequence of another state, i.e., how a specific state may evolve into another state. The detailed properties and structure of a state will not be of interest for the purpose of this paper. The state encompasses state components for whatever the laws of physics require.

The laws of physics allow us to derive from a given state $S_{0}$ a sequence of states $S_{1}, S_{2}, \ldots, S_{n}$ such that it is said $S_{j}$ is caused by $S_{i}$ if $j>i .{ }^{4}$

In general, the (causal) laws of physics support the computation of a state $S_{j}$ given a state $S_{i}$ with $j>i$.

The computation typically requires a parameter "timedifference" $\Delta$ t between states $S_{i}$ and $S_{j}$

(1) $\mathrm{S}_{\mathrm{j}}=\operatorname{applyLawsOfPhysics}\left(\mathrm{S}_{\mathrm{i}}, \Delta \mathrm{t}\right), \mathrm{j}>\mathrm{i}$.

The parameter $\Delta \mathrm{t}$ (time-difference) may be considered as specifying the distance within the causal sequence of states, e.g., $\Delta \mathrm{t}=\mathrm{j}$ - $\mathrm{i}$.

In general, the laws of physics can also be used to compute a state $S_{i}$ from $S_{j}$, where $i<j$ (i.e., backwards in the causal chain of states)

(2) $\mathrm{S}_{\mathrm{i}}=$ applyLawsOfPhysicsBW $\left(\mathrm{S}_{\mathrm{j}}, \Delta \mathrm{t}\right)$.

Notice the symmetry as well as the asymmetry expressed in equations (1) and (2). The symmetry can be

\footnotetext{
${ }^{2}$ In [13] I. Stewart distinguishes the symmetry of the equations of physics from the (a-)symmetry of the solutions.

3 Allowing for indeterminism makes the argument a little more complicated but does not lead to different conclusions.

${ }^{4}$ In [4] causality is also explained by a (partially) ordered set of states, similar, but not identical, to what is here described.
}

observed by the fact that both computations, the "forward computation" and the "backward computation", are based on the same laws of physics. The asymmetry lies in the fact that the functions representing forward computation and the backward computation, applyLawsOfPhysics() and applyLawsOfPhysicsBW(), although both belonging to the same set of laws, in general are not identical (applyLawsOfPhysics( ) $\neq$ applyLawsOfPhysicsBW() ). The asymmetry can be hidden by defining

$\mathrm{S}^{\prime}=$ applyLawsOfPhysics $(\mathrm{S}, \Delta \mathrm{t}) \equiv \mathrm{S}^{\prime}=$ applyLawsOfPhysicsBW( $\mathrm{S}, \Delta \mathrm{t})$ and stating that the parameter $\Delta \mathrm{t}$ must be positive if $\mathrm{S}^{\prime}$ is caused by $\mathrm{S}$ and negative if $\mathrm{S}$ is caused by $\mathrm{S}^{\prime}$. However, this merely shifts the asymmetry to the application instructions for the function.

\subsection{Time}

The concept of time enters our discussion via the parameter $\Delta \mathrm{t}$, which appears above within applyLawsOfPhysics $\left(\mathrm{S}_{\mathrm{i}}, \Delta \mathrm{t}\right)$. However, while in the above equations $\Delta \mathrm{t}$ simply specifies a distance within the causal sequence of states, time plays a much more fundamental role within the laws of physics. The time coordinate is part of the states that constitute the world, or conversely, one may view states as being assigned to space-time coordinates. The simplest way to include the time coordinate with the states would be to derive the time coordinate directly from the time-difference $\Delta \mathrm{t}$ used during the computation of new states. ${ }^{5}$

Relativity theory tells us that we have to distinguish (1) the (global) coordinate time and (2) the time as experienced by a local system, the so-called proper time (or wristwatch time). Because our discussion on causality is based on global states, it is reasonable (though not mandatory) to make the time-difference $\Delta \mathrm{t}$ and the time coordinate value refer to the global coordinate time. ${ }^{6}$

\subsection{Time Arrow}

With the above description, the asymmetry within the application of the laws of physics is seen in the distinction between the "forward computation" with applyLawsOfPhysics $\left(\mathrm{S}_{\mathrm{i}}, \Delta \mathrm{t}\right)$ (or $\Delta \mathrm{t}>0$ ) and the "backward computation" with applyLawsOfPhysicsBW( $\left.\mathrm{S}_{\mathrm{j}}, \Delta \mathrm{t}\right)$, (or $\Delta \mathrm{t}$ $<0$ ). This may be considered as a direction of time or a time arrow due to the causal laws followed by nature.

\section{The World's Dynamical Evolution Process}

The collection of functions (equations and axioms) that make up the theories of physics do not automatically imply the complete description/understanding of the overall process of the world's dynamical evolution.

Let us view the laws for the evolution of the state of the world (i.e., the laws of physics) as represented by a "physics-interpreter". The physics-interpreter acts upon the state of the world.

\footnotetext{
${ }^{5}$ In practice, it works the other way around - $\Delta$ t is determined as the difference of the time coordinates.

${ }^{6}$ This makes the function applyLawsOfPhysics $\left(S_{i}, \Delta t\right)$ a little more complicated, because most laws of physics refer to the proper time.
} 
To show the dynamical evolution, the model described here assumes the continuous repeated invocation of the physics-interpreter to realize the progression of the state of the world. The physics-interpreter continuously determines new states. The physics-interpreter acts upon an in-world to generate an out-world.

worldEvolution( world W ) :=

$\mathrm{W} . \mathrm{t}=0 ; \mathrm{W} \cdot \mathrm{x}_{1}=0 ; \mathrm{W} \cdot \mathrm{x}_{2}=0 ; \mathrm{W} \cdot \mathrm{x}_{3}=0$;

$\mathrm{W} \cdot \psi=$ initialState;

$\Delta \mathrm{t}=$ timestep; // must be positiv //

DO UNTIL ( nonContinueState( W ) ) \{ \}

physicsInterpreter $(\mathrm{W}, \Delta \mathrm{t})$;

\}

physicsInterpreter $(\mathrm{W}, \Delta \mathrm{t}):=\{$

tdt $=W . t+\Delta t$;

$\mathrm{W}=\operatorname{applyLawsOfPhysics}(\mathrm{W}, \Delta \mathrm{t})$;

discardSpacetimePointsWithTimeCoordinate(W.t $<$ tdt ); \}

with state parameters

world := \{ spacetimepoint ... $\}$

spacetimepoint $:=\left\{t, x_{1}, x_{2}, x_{3}, \psi\right\}$

$\psi:=\left\{\right.$ stateParameter $_{1}, \ldots$, stateParameter $\left.{ }_{n}\right\}$

Of particular interest for the purposes of this paper are the possible changes in space-time with respect to the time component. In line with the description in section 4 , the following rules with respect to the time coordinate of space-time are proposed for the application of the physicsinterpreter:

- Rule1: The time coordinate of the space-time points belonging to the out-world is uniformly equal to the time coordinate of the in-world increased by some constant amount (e.g., 1).

- Rule2: Given that the in-world has consistently older time-coordinates than the out-world, a further result of applying the physics-interpreter to an in-world is that the in-world is discarded and replaced by the generated out-world.

The above two rules (or similar ones) are crucial in discussing not only the possible existence of a time arrow, but also causality and concepts for the progression of the world state in general.

Rule1's proclamation that the time coordinate is always increased by a constant amount may be interpreted as defining a direction of time. Replacement of Rule1 by a rule that is time symmetric would seem rather arbitrary.

\section{The Observation of Time Asymmetry}

From a philosophical point of view, one may argue that an arrow of time exists if and only if it is possible, in principle, to observe its existence. The standard example used in the literature for testing the existence of an arrow of time is the movie of some physical process (for example, an egg falling from a table and breaking) running backwards. It is argued that if it is not possible to detect that the movie is running backwards, this is an indication or proof that there exists no arrow of time for that particular process.

The author claims that such a "movie test" can be accepted as a proof for the non-existence of an arrow of time for that particular process only if two conditions are satisfied
1. The initial state configuration of the process is known completely and taken into consideration.

2. The information about the dynamical behaviour of the process is not restricted to a movie but must include all further relevant information. ${ }^{7}$

Within the literature discussing the possible existence of a time arrow, it is generally requirement (1) that is ignored. For example, for a movie showing the trajectories of scattered billiard-balls it may not be observable whether the movie is running forward or backward as long as no information on the initial state (e.g., the initial momenta of the balls) is given. As soon as the information on the initial state is provided, there is no question about the direction of the movie (and the direction of a time arrow).

The author claims that, when the above-mentioned complete information is available, it will be possible to observe a time arrow, even when entropy effects are ignored. Of course, it will be possible to construct experiments where no arrow of time can be observed. However, such an experiment cannot prove the general absence of a time arrow.

Let us assume that an agreement can be reached on the general existence of an arrow of time and that the evolution of the state of the world functions as described in section 5. It may then still be reasonable to perform further experiments to check for possible deviations from the assumed general dynamical behaviour. For example, it may be suspected that in the neighbourhood of a black hole, deviations from the known causal laws of physics might be found. If such deviations can be found, this would likely not require the rejection of an arrow of time but rather, a modification of some specific theory. For example, in quantum field theory, a popular interpretation of antiparticles is that these particles are travelling backwards in time (see [10], page 638).

\section{Discussion}

\subsection{Global States and Time Coordinate}

The rules stated in sections 4 . and 5. refer to a global state encompassing the world.

The assumption of a global state leads to questions about the time coordinates that may need to be assigned to the state as a whole or to state components. Theories about the overall evolution of the universe tend to assume global states and global time coordinates (see, for example [1] and [12]). With reference to Barbours' work [1], H.D. Zeh writes in [15], page 168, 'Barbours' essential assumption of global states in the sense of simultaneousness seems to be a relict from his non-relativistic theory. A relativistic theory should replace the global "Now" by "Here and Now". The same comment may be made about the global state assumed in this paper.

Although it is true that relativity theory has dropped the concept of "global simultaneousness", this does not disallow the idea of a progression of the state of the world by common, unique time steps. Local (proper) time coordinates alone are not sufficient for the definition of a

\footnotetext{
${ }^{7}$ A more complete discussion of this requirement needs some refinement as to what "relevant information" means. The processes of quantum physics imposing limitations on concurrently observable information may also require repetition of the observation experiments.
} 
global state and the representation of space-time trajectories. In cases when trajectories meet at a common point in space-time, simultaneousness is trivial, while the continuation by a common local time coordinate as a substitute for a global time coordinate is not feasible.

A possible definition and identification of a global time coordinate does not need to be in conflict with the relational simultaneousness introduced by special relativity. The definition could refer to a time base such as, for example, the time associated with the aging of the cosmic background radiation.

A second question then is whether it is reasonable to assume the progression of the state of the world to occur in uniform global time steps. The concept described in the preceding sections could be easily adapted to (uniform) local time steps. In that case the progression of the state of the world by global time steps would have to be distributed to the progression of the individual inertial systems with differing amounts of local time steps.

\subsection{Entropy and Time Arrow}

This paper starts with the authors claim that the entropy law might not be a suitable base for the establishment of an arrow of time. There exist several different versions of the formulation of the entropy law (see [7]). The original version, the second law of thermodynamics, was formulated by Rudolph Clausius in 1850. Attempts have been made to generalize the definition of entropy as a measure of the irreversibility of a quasistatic process. Also, formulations exist where entropy is seen as a measure for the ordering of the state of a system. These more generalized versions of the entropy law are based on considerations of the state of a physical system and of the evolution of the state in accordance with the laws of physics.

The topics laws of physics and state of a system are also addressed in this paper (sections 3, 4, and 5) with the conclusion that the function

$\mathrm{S}_{\mathrm{j}}=\operatorname{applyLawsOfPhysics}\left(\mathrm{S}_{\mathrm{i}}, \mathrm{t}\right)$ is not time symmetrical if its application to the world's dynamical evolution process is considered. This raises the question whether these considerations also apply to the "state-based" formulations of the entropy law.

The author claims that the "state-based" formulations of the entropy law also assume a non-symmetrical application of $\mathrm{S}_{\mathrm{j}}=$ applyLawsOfPhysics $\left(\mathrm{S}_{\mathrm{i}}, \Delta \mathrm{t}\right.$ ) (i.e., always assume the "forward computation" only). This may be viewed as an argument that the entropy law (at least the generalized state-based versions) presupposes an arrow of time rather than that an arrow of time can be derived from the entropy law.

\section{Conclusions}

The major conclusion of this paper is that one does not need the effects of increasing entropy to determine an arrow of time for the dynamical evolution of the world.

After in section 2 the validity of the standard argument for the negation of a direction of time (i.e., the apparent symmetry of the laws of physics under a reversal of the direction of time) is shown to be questionable, this paper investigated the possible determination of an arrow of time. In the preceding sections, three arguments were identified for the existence of an arrow of time:

1. Causality can be derived from the laws of physics and causality implies a direction of time. (section 4).

2. A reasonable model of the overall dynamical evolution process of the world implies a direction of time (section 5).

3. The arrow of time is observable if all relevant information is included in the observation (see section 6).

Findings (1) and (2) cannot be deduced from entropy considerations and are in conflict with the claimed time symmetry of the laws of physics. Section 2 explains that the apparent time symmetry of the laws of physics is a (valuable) static property. The symmetry disappears (i.e., is broken) when the dynamic application of the laws of physics by nature is considered (as described in sections 4 and 5).

The conclusions expressed in (1) and (2) are based on various overall assumptions with respect to the existence of a global state of the world and the overall process for the progression of the state of the world. These assumptions seem to be implicit assumptions within various areas of physics. However, they have not yet been stated explicitly (to the author's knowledge). Although the assumptions have been developed in the context of the subject of this paper, the author believes that the explicit formulation of these (or of alternative ones) is highly desirable within physics in general.

\section{References}

[1] Barbour J. The End of Time. Oxford University Press, 2001.

[2] Basieux P. Die Architektur der Mathematik. Rowohlt Taschenbuch Verlag, Hamburg, 2000.

[3] Everett H. III Relative State' formulation of Quantum Mechanics. Rev Mod Phys 29, 454, 1957

[4] Frisch M. Causal Models and the Asymmetry of State Preparation. in EPSA Philosophical Issues in the Sciences, vol.2, eds. M. Suárez, M. Dorato, and M. Redei, 2010.

[5] Frisch M. Laws in Physics. European Review, Volume 22, pp S33-S49, 2014.

[6] Horwich P. Asymmetries in Time. MIT Press, Cambridge Ma, 1987.

[7] Lemons D S. A Student's Guide to Entropy. Cambridge University Press, Cambridge UK, 2013.

[8] Maudlin T. Philosophy of Physics - Space and Time. Princeton University Press, 2012.

[9] Maudlin T. Three Measurement Problems. Topoi-Int Rev Philos 14, 1995.

[10] Penrose R. The Road to Reality. Vintage Books, New York, 2005.

[11] Price H. Time's and Archimedes' Point. Oxford University Press, 1996.

[12] Smolin L Time Reborn. From the Crisis in Physics to the Future of the Universe. Houghton Mifflin Harcourt, New York, 2013.

[13] Stewart I Seventeen Equations that Changed the World. Profile Books, London, 2012.

[14] Weinberg S. The Quantum Theory of Fields, Volume 1, Foundations. Cambridge University Press, 2005.

[15] Zeh H D. Physik ohne Realitaet: Tiefsinn oder Wahnsinn. Springer Verlag, Heidelberg, 2012. 